\title{
Experimenting with Apéry Limits and WZ pairs
}

\author{
ROBERT DOUGHERTY-BLISS and DORON ZEILBERGER, Department of Mathematics, Rutgers
} University (New Brunswick), Hill Center-Busch Campus, 110 Frelinghuysen Rd., USA

\begin{abstract}
This article, dedicated with admiration in memory of Jon and Peter Borwein, illustrates by example, the power of experimental mathematics, so dear to them both, by experimenting with so-called Apéry limits and WZ pairs. In particular we prove a weaker form of an intriguing conjecture of Marc Chamberland and Armin Straub (in an article dedicated to Jon Borwein), and generate lots of new Apéry limits. We also rediscovered an infinite family of cubic irrationalities, that suggested very good effective irrationality measures (lower than Liouville's generic 3), and that we conjectured to go down to the optimal 2. As it turned out, as pointed out by Paul Voutier (see the postscript kindly written by him), our conjectures follow from deep results in number theory. Nevertheless we believe that further experiments with our Maple programs would lead to new and interesting results.
\end{abstract}

\section{Recommended Reference Format:}

Robert Dougherty-Bliss and Doron Zeilberger. 2021. Experimenting with Apéry Limits and WZ pairs . Maple Trans. 1, 2, Article 14359 (November 2021), 9 pages. https://doi.org/10.5206/mt.v1i2.14359

In fond memory of the amazing Borwein brothers: Fonathan (20 May 1951 - 2 August 2016) and Peter (10 May 1953 - 23 August 2020), great pioneers of Experimental Mathematics

\section{Preface: The amazing Borwein Brothers}

[This section was written by DZ, hence the first-person "I"]

I first met the Borwein brothers in the historic Centenary Ramanujan conference, at the University of Illinois at Urbana-Champaign, that took place June 1-5, 1987. They were already then legendary. They just published the classic [BoBo1] and their talk [BoBo2] was a natural continuation of their great book, furnishing yet-faster-converging series for computing $\pi$, inspired by the work of Ramanujan. Here is the conference photo:

https://sites.math.rutgers.edu/ zeilberg/mamarim/mamarimhtml/Ramanujan100.jpg

You can see Jon Borwein in the front row (6th from the right, sporting a Pi T-shirt), and Peter Borwein in the fourth row ( 7 th from the left). Of course I was in awe of them, and they complemented the other notable $\pi$-brothers, Gregory and David Chudnowsky (who were present, but are not in the photo).

Since then, Jon and Peter became pioneers in Experimental Mathematics. They founded the Center for Constructive and Experimental Mathematics (CECM) at Simon Fraser University http://www.cecm.sfu.ca/, which is still flourishing today.

Then Jon, the epitome of type-A personality, always with so many plans, went down-under and founded CARMA (COMPUTER-ASSISTED RESEARCH MATHEMATICS AND ITS APPLICATIONS), https://carma.newcastle.edu.au/about/, and this center is just as flourishing today.

Together with David Bailey, Jon wrote the bible of Experimental Mathematics [BoBa]. This was complemented by a second volume (where Ronald Girgensohn joined them) [BoBaGi]. These two

Authors' address: Robert Dougherty-Bliss, robert.w.blissatgmaildotcom; Doron Zeilberger, DoronZeilatgmaildotcom, Department of Mathematics, Rutgers University (New Brunswick), Hill Center-Busch Campus, 110 Frelinghuysen Rd., Piscataway, NJ, USA, 08854-8019.

Permission to make digital or hard copies of all or part of this work for any use is granted without fee, provided that copies bear this notice and the full citation on the first page. Copyrights for third-party components of this work must be honored. (c) 2021 Copyright held by the owner/author(s). Publication rights licensed to Maple Transactions, under Creative Commons CC-BY 4.0 License.

https://doi.org/10.5206/mt.v1i2.14359

Maple Trans., Vol. 1, No. 2, Article 14359. Publication date: November 2021. 
volumes are so engaging, yet very deep. See my raving book review for American Scientist [Ze3]: https://sites.math.rutgers.edu/ zeilberg/mamarim/mamarimPDF/mathexp.pdf.

Note that, defying the almost universal mathematical tradition of naming the authors alphabetically, Jon Borwein is listed as first author in these two volumes. I am sure that it is for a very good reason!

I should also mention that both Jon and Peter did so much to cultivate the $\pi$-cult, and the anthology $[\mathrm{BerBoBo}]$ is an indispensible source for all $\pi$-lovers.

\section{The present article: A continuation of Section 10.3 of the Borweins' masterpiece "Pi and the AGM"}

The present article is inspired by the work of Roger Apéry [Ap] and Frits Beukers [Beu], beautifully exposited in Pi and the AGM, Section 10.3. It also continues the work in [Ze2], and the recent delightful article, by Marc Chamberland and Armin Straub [ChaS], that was dedicated to the memory of Jon Borwein.

\subsection{Apéry Limits}

One way that Apéry's seminal proof of the irrationality of $\zeta(3)$ could have been discovered, in a counterfactual world, was to consider, out of the blue, the second-order recurrence

$$
n^{3} u_{n}-\left(17 n^{2}+51 n+39\right)(2 n+3) u_{n-1}+(n-1)^{3} u_{n-2}=0,
$$

and let $a_{n}$ and $b_{n}$ be the solutions of that recurrence with initial conditions

$$
a_{0}=0, a_{1}=6 \quad ; \quad b_{0}=1, b_{1}=5,
$$

then let the computer compute many terms, evaluate $\frac{a_{1000}}{b_{1000}}$ to many decimals, and then use Maple's identify, and lo and behold, get that it (most probably) equals $\zeta(3)$ (i.e. $\sum_{i=1}^{\infty} \frac{1}{i^{3}}$ ). Then, still empirically and numerically, after rewriting $\frac{a_{n}}{b_{n}}$ as $\frac{a_{n}^{\prime}}{b_{n}^{\prime}}$, where now both numerator and denominators are integers (initially $b_{n}$ are integers, but $a_{n}$ are not), estimate that there exists a positive number $\delta$ (about 0.0805) such that

$$
\left|\frac{a_{n}^{\prime}}{b_{n}^{\prime}}-\zeta(3)\right| \leq \frac{\text { CONSTANT }}{\left(b_{n}^{\prime}\right)^{1+\delta}},
$$

that immediately entails (see $[\mathrm{vdP}])$ that $\zeta(3)$ is irrational.

This theme is pursued in [Ze2] and much more recently in [DKZ], where the motivation was to discover irrationality proofs of other constants.

In the above-mentioned delightful article [ChaS], the authors decided to study Apéry limits independently of their potential for suggesting irrationality proofs, continuing the work of Zudilin $[\mathrm{Zu}]$ and Almkvist, van Straten and Zudilin [AlvSZ]. Take any linear recurrence, that came up 'naturally' (e.g. satisfied by a binomial coefficients sum), then define another solution using different initial conditions, and take the limit of the ratios of the two sequences, and see what happens.

In particular, they made the following intriguing conjecture (Conjecture 9 in [ChaS]).

Conjecture (Chamberland and Straub) For $d \geq 3$, the minimal order recurrence satisfied by

$$
A^{(d)}(n):=\sum_{k=0}^{n}\left(\begin{array}{l}
n \\
k
\end{array}\right)^{d},
$$

has a unique solution, $B^{(d)}(n)$, with $B^{(d)}(0)=0$ and $B^{(d)}(1)=1$ that has the property that:

$$
\lim _{n \rightarrow \infty} \frac{B^{(d)}(n)}{A^{(d)}(n)}=\frac{\zeta(2)}{d+1} .
$$


Surprisingly, Chamberland and Straub did not address the rate of convergence. After all, the original motivation of Apéry was to furnish sequences of rational numbers converging to the desired constant $(\zeta(3)$ or $\zeta(2))$ with exponentially decaying rate. In fact he needed more than that (and he lucked out), that the denominators do not grow too fast, but setting this number-theoretical issue aside, even if our sequence of rational numbers does not imply irrationality, it is desirable to demand that the error in the approximation decays exponentially.

It is easy to see that if the Chamberland-Straub conjecture is true, then the following stronger (and far more interesting!) version is also true. Hence their convergence is equivalent to

Stronger Chamberland-Straub Conjecture For $d \geq 3$, the minimal order recurrence satisfied by

$$
A^{(d)}(n):=\sum_{k=0}^{n}\left(\begin{array}{l}
n \\
k
\end{array}\right)^{d},
$$

has a unique solution, $B^{(d)}(n)$, with $B^{(d)}(0)=0$ and $B^{(d)}(1)=1$ that has the property that there exists real numbers $C$ and $\alpha>1$ such that

$$
\lim _{n \rightarrow \infty}\left|\frac{B^{(d)}(n)}{A^{(d)}(n)}-\frac{\zeta(2)}{d+1}\right| \leq \frac{C}{\alpha^{n}}
$$

Using the results in [Ze1] we can prove a weaker form of this stronger conjecture.

Theorem 1: There exists some (not necessarily minimal) recurrence and some initial conditions for the $B^{(d)}(n)$ sequence such that both $A^{(d)}(n)$ and $B^{(d)}(n)$ satisfy the same linear recurrence equation with polynomial coefficients and real numbers $C$ and $\alpha>1$ such that

$$
\lim _{n \rightarrow \infty}\left|\frac{B^{(d)}(n)}{A^{(d)}(n)}-\frac{\zeta(2)}{d+1}\right| \leq \frac{C}{\alpha^{n}}
$$

In order to prove this, we need to recall the following theorem from [Ze1]. Below $N$ is the forward shift operator, and a general linear recurrence operator, of order $L$, say, has the form

$$
\sum_{i=0}^{L} c_{i}(n) N^{i}
$$

so another way of saying that a sequence $a(n)$ satisfies the linear recurrence equation

$$
\sum_{i=0}^{L} c_{i}(n) a(n+i)=0,
$$

is to say that the sequence $a(n)$ is annihilated by the operator $\sum_{i=0}^{L} c_{i}(n) N^{i}$, i.e.

$$
\left(\sum_{i=0}^{L} c_{i}(n) N^{i}\right) a(n)=0 \text {. }
$$

We need the following theorem.

Theorem 2 (Theorem 9 of [Ze1]): Let $c(n, k)$ be the potential function of a WZ 1-form $F(n, k) \delta k+$ $G(n, k) \delta n$ in the two variables $(n, k)$. In other words,

$$
F(n, k)=c(n, k+1)-c(n, k), G(n, k)=c(n+1, k)-c(n, k),
$$

and let $b(n, k)$ be closed-form (i.e. 'proper-hypergeometric'). Let

$$
a(n):=\sum_{k=0}^{n} c(n, k) b(n, k), \quad b(n):=\sum_{k=0}^{n} b(n, k) .
$$


There exist (rapidly exhibitable) linear recurrence operators with polynomial coefficients $R(N, n)$ and $S(N, n)$ such that

$$
R(N, n) b(n)=0, \quad S(N, n) R(N, n) a(n)=0 .
$$

Furthermore, there exist rapidly exhibitable closed-form "certificates" $B(n, k)$ and $D(n, k)$ such that the following routinely verifiable identities are true:

$$
R(N, n) b(n, k)=B(n, k)-B(n, k-1),
$$

$S(N, n) R(N, n)(b(n, k) c(n, k))=S(N, n)(c(n, k) B(n, k)-c(n, k-1) B(n, k-1))+D(n, k)-D(n, k-1)$.

In addition, $B(n, k) / b(n, k)$ and $D(n, k) /(b(n, k) F(n, k))$ are both rational functions.

Proof of Theorem 1. Consider the potential function, let's call it $c(n, k)$

$$
c(n, k):=2 \sum_{m=1}^{n} \frac{(-1)^{m-1}}{m^{2}}+\sum_{m=1}^{k} \frac{(-1)^{n+m-1}}{m^{2}\left(\begin{array}{c}
n \\
m
\end{array}\right)\left(\begin{array}{c}
n+m \\
m
\end{array}\right)},
$$

that comes from the WZ form

$$
\omega_{\zeta(2)}:=\frac{(-1)^{(n+k)} k !^{2}(n-k-1) !}{(n+1)(n+k+1) !}[(n+1) \delta k+2(n-k) \delta n]
$$

(see [Ze1]), then it follows from Theorem 2, that for any binomial coefficients sum ,

$$
A(n):=\sum_{k=0}^{n} b(n, k) \text {, }
$$

(not just powers of $\left(\begin{array}{l}n \\ k\end{array}\right)$ ), defining

$$
B(n)=\sum_{k=0}^{n} b(n, k) c(n, k)
$$

that there exists a linear recurrence satisfied by both sequences $A(n)$ and $B(n)$.

Since $c(n, k)$ converges to $\zeta(2)$ no matter how you approach $(\infty, \infty)$ on the discrete region $\{n \geq k \geq 0\}, B(n) / A(n)$, being a weighted-average of $c(n, k)$ for $0 \leq k \leq n$, also must converge to $\zeta(2)$.

It also follows from the proof of Theorem 2 in [Ze1] that the convergence rate is exponentially decaying.

Comment: Armin Straub noticed that there exist more straightforward ways to prove Theorem 1, but we believe that our construction has the potential to yield better irrationality measures.

One of our dreams is to find a decision procedure that:

Inputs: An arbitrary linear recurrence equation with polynomial coefficients of order $L$, say, and any two solutions given by some initial conditions,

$$
\begin{aligned}
& a(0)=a_{0}, \quad \ldots \quad, \quad a(L-1)=a_{L-1}, \\
& b(0)=b_{0}, \quad \ldots, \quad b(L-1)=b_{L-1},
\end{aligned}
$$

Outputs: Yes if and only if $\lim _{n \rightarrow \infty} \frac{a(n)}{b(n)}=0$.

This decision procedure exists for constant coefficients linear recurrences (it goes back at least to Euler), but we have no clue how to do it for linear recurrences with polynomial coefficients. 
Assuming that we have such an 'oracle', it would be immediate to prove the Chamberland-Straub conjecture for any specific $d$. Both $A^{(d)}(n)$ and $B^{(d)}(n)$ satisfy the same minimal recurrence of $A^{(d)}(n)$. Let

$$
B^{(d)}(n)=\sum_{k=0}^{n}\left(\begin{array}{l}
n \\
k
\end{array}\right)^{r} c(n, k)
$$

then, by linearity, $B^{(d)}(n)-B^{\prime(d)}(n)$ also satisfies that very same (non-minimal recurrence), and if we can rigorously decide whether or not

$$
\lim _{n \rightarrow \infty} \frac{B^{(d)}(n)-B^{(d)}(n)}{A^{(d)}(n)}=0,
$$

we would be able to completely prove the Chamberland-Straub conjecture (for each specific power $d$ ).

Since we do not (yet!) have such a decision procedure, we can do the next-best thing and prove it empirically. This is done for $3 \leq d \leq 9$ in the output file

https://sites.math.rutgers.edu/ zeilberg/tokhniot/oAperyWZnew1.txt ,

where the (rigorous!) proof of the weaker form of the Chamberland-Straub conjecture is given, followed by a non-rigorous, 'empirical proof' of the full conjecture.

\section{Having fun with Apéry Limits}

"Apéry's incredible proof appears to be a mixture of miracles and mysteries. The dominating question is how to generalise all this, down to the Euler constant $\gamma$ and up to the general $\zeta(t)$ ?." [vdP, sec. 10]

The intervening forty years have not answered van der Poorten's question; we still do not know how to construct a "nice" recurrence to prove the irrationality of fixed constants. However, as mentioned in [ChaS], the inverse problem is just as difficult! That is, given solutions $A(n)$ and $B(n)$ to some recurrence with polynomial coefficients, if

$$
\lim _{n \rightarrow \infty} \frac{B(n)}{A(n)}
$$

exists, what does it equal? (The same problem for recurrences with constant coefficients is much easier.)

The conjecture of [ChaS] considered in the previous section was made with numerical evidence. In some sense this conjecture was "easy" because most computer algebra systems can identify $r \zeta(k)$ for reasonably simple rationals $r$ and small $k$. However, for an arbitrary Apéry $\operatorname{limit}_{\lim _{n \rightarrow \infty} B(n) / A(n)}$ we are usually stranded.

To help experiment, the Maple package

https://sites.math.rutgers.edu/ zeilberg/tokhniot/AperyLimits.txt

systematically searches for recurrences and attempts to guess the corresponding Apéry limits using our enhanced version of Maple's ident i fy command. The recurrences come from either the Zeilberger algorithm [Ze0] or from the Almkvist-Zeilberger algorithm [AlZ].

For example, consider the sequence of functions

$$
f_{n}(x)=\left(\frac{(2 x+1)(1+3 x)}{x}\right)^{n} x^{-2 / 3}
$$

The Almkvist-Zeilberger algorithm shows that the sequence of contour integrals

$$
a(n)=\int_{|z|=1} f_{n}(z) d z
$$


satisfies the recurrence

$$
a(n)=9 n \frac{(2 n-1) 5}{(3 n-1)(3 n+1)} a(n-1)-9 \frac{n(n-1)}{(3 n-1)(3 n+1)} a(n-2) .
$$

We will now forget $a(n)$ itself and make use only of the recurrence. Let $A(n)$ and $B(n)$ satisfy this same recurrence with initial conditions

$$
A(0)=1, \quad A(1)=0 ; \quad B(0)=0, \quad B(1)=1 .
$$

Then, empirically,

$$
\lim _{n \rightarrow \infty} \frac{B(n)}{A(n)}=-\frac{28}{15} 18^{1 / 3}-\frac{32}{45} 18^{2 / 3}-\frac{76}{15} .
$$

Going through the same process with

$$
f_{n}(x)=\left(\frac{(3 x+1)(1+4 x)}{x}\right)^{n} x^{-2 / 3}
$$

gives an empirical limit of

$$
-\frac{80}{21} 6^{1 / 3}-\frac{44}{21} 6^{2 / 3}-\frac{148}{21}
$$

Both sequences are instances of the more general family

$$
f_{n}(x)=\left(\frac{(c x+1)(1+(c+1) x)}{x}\right)^{n} x^{-2 / 3}
$$

Indeed, going through the same steps with arbitrary $c$ suggests that we always obtain a "cubic" Apéry limit. In particular, we conjectured that the induced Apéry limit is the real root of the cubic

$$
64+144 x(1+2 c)+108 x^{2}\left(3 c^{2}+3 c+1\right)+27 x^{3}(1+2 c)=0
$$

which we determined thanks to the PSLQ algorithm [BaFe].

Even better than the Apéry limit itself, as $c \rightarrow \infty$, it seems that the effective irrationality measures suggested by our computations go down to 2 . Recall that for a cubic irrationality, the 'vanilla' irrationality measure promised by Liouville is 3 , so any 'infinite family' of cubic irrationalities that give irrationality measures smaller than 3 is of interest. Such families were found by Alan Baker [Bak], Gregory Chudnowsky [Chu], Michael Bennett [Be], and Paul Voutier [V1][V2].

As it turned out, our conjectures follow from known deep results in number theory, see the postscript kindly written by Paul Voutier. But this was just one example, and hopefully further experiments would lead to new results.

For a computer-generated exploration of this experiment, see the output file

https://sites.math.rutgers.edu/ zeilberg/tokhniot/oAperyLimits5.txt .

Similar to this infinite family of cubic irrationalities, the sequence of functions

$$
f_{n}(x)=\left(\frac{(c x+1)(1+(c+1) x)}{x}\right)^{n} x^{-1 / 2} .
$$

empirically produces the quadratic Apéry limit

$$
-3 c-\frac{3}{2}-3 \sqrt{c^{2}+c}
$$

Every quadratic irrationality has an effective irrationality measure of 2 (from its continued fraction expansion), but our diophantine approximations are different from the standard continued fraction continuants. This is all conjecture, but we are sure that it won't be too hard to prove it. Since the stakes are so low, and we are experimental mathematicians, we leave this to the reader.

For a computer-generated discussion of this experiment, see the file 


\section{https://sites.math.rutgers.edu/ zeilberg/tokhniot/oAperyLimits6.txt}

Alas, most constants that we were able to identify are known to be irrational by general theorems (e.g. the Lindemann-Weierstrass theorem). For those unidentified constants our results may suggest explicit irrationality measures, and for the identified constants they may suggest effective irrationality measures.

\section{Postscript by Paul Voutier}

The authors conjecture that the induced Apéry limit is the real root, $\alpha_{c}$, of the cubic polynomial in $x$

$$
f_{c}(x)=64+144 x(1+2 c)+108 x^{2}\left(3 c^{2}+3 c+1\right)+27 x^{3}(1+2 c)=0
$$

and claim that as $c \rightarrow \infty$, it seems that the effective irrationality measures suggested by their computations go down to 2 .

We show here that their suggestion is true. A new such family of algebraic numbers with effective irrationality measures improving on Liouville's measure would be of considerable interest. But $\alpha_{c}$ is in $Q\left(((c+1) / c)^{1 / 3}\right)$ and effective irrationality measures for elements of such fields have been known since Baker's pioneering work in 1964 [Bak]. This was later improved by Chudnowsky [Chu], with explicit versions of Chudnowsky's work produced by Easton [E], Bennett [Ben] and Voutier [V1].

Using Maple, one can quickly verify that

$$
\frac{4((c+1) / c)^{1 / 3}-4}{-(9 c+3)((c+1) / c)^{1 / 3}+9 c+6}
$$

is a root of $f_{c}(x)$ and, since real when taking the real cube root of $(c+1) / c$, it must equal $\alpha_{c}$.

Using Theorem 2.1 of [V1] with $a=c+1$ and $b=c$, we can take $d=0$ there, and so

$$
\kappa=\frac{\log \left(e^{0.911}(\sqrt{c+1}+\sqrt{c})^{2}\right)}{\log \left(e^{-0.911}(\sqrt{c+1}-\sqrt{c})^{-2}\right)} .
$$

For $c \geq 4$, we have $\kappa<2$. Thus,

$$
\left|((c+1) / c)^{1 / 3}-p / q\right|>\frac{1}{10^{120}(c+1)|q|^{\kappa+1}}
$$

for all integers $p$ and $q$ with $q \neq 0$, which improves on Liouville's irrationality measure for such $c$.

Since $\alpha_{c}$ is a fractional linear transformation of $((c+1) / c)^{1 / 3}$ with rational coefficients, we can use Lemma 6.3 of [V2] to obtain an effective irrationality measure for $\alpha_{c}$ too. In the notation of Lemma 6.3 of [V2], we have $a_{1}=4, a_{2}=-4, a_{3}=-(9 c+3), a_{4}=9 c+6$ with $\theta=((c+1) / c)^{1 / 3}$ and $\theta^{\prime}=\alpha_{c}$. For $c \geq 4$, it can be shown that

$$
\left|a_{4}+a_{3} \theta\right|\left(\left|a_{3}\right|\left(1+\left|\theta^{\prime}\right|\right)+\left|a_{1}\right|\right)^{\kappa}<\left|a_{4}+a_{3} \theta\right|\left(\left|a_{3}\right|\left(1+\left|\theta^{\prime}\right|\right)+\left|a_{1}\right|\right)^{2}<1000 c^{2},
$$

so

$$
\left|\alpha_{c}-p / q\right|>\frac{1}{10^{123}(c+1)^{3}|q|^{\kappa+1}}
$$

for all integers $p$ and $q$ with $q \neq 0$.

Furthermore, as $c \rightarrow \infty$, we have

$$
\kappa \rightarrow \frac{\log \left(e^{0.911} 4 c\right)}{\log \left(e^{-0.911} c / 4\right)} \rightarrow 1,
$$


so Dougherty-Bliss and Zeilberger's observation that the effective irrationality measures approach 2 as $c \rightarrow \infty$ is true.

Acknowledgment: Many thanks are due to Michael Bennett and Wadim Zudilin for very helpful discussions. Also many thanks to Armin Straub for very helpful comments on an earlier version, and to Paul Voutier who authored the postscript.

\section{REFERENCES}

[AlZ] Gert Almkvist and Doron Zeilberger, The method of integrating under the integral sign, J. Symbolic Comp. 10 (1990), 571-591. https://sites.math.rutgers.edu/ Zeilberg/mamarim/mamarimPDF/ duis.pdf

[AlvSZ] Gert Almkvist, Duco van Straten, and Wadim Zudilin, Apéry limits of differential equations of orders 4 and 5, Fields Inst. Commun. Ser. 54 (2008), 105-123.

[Ap] Roger Apéry, "Interpolation de fractions continues et irrationalité de certaine constantes" Bulletin de la section des sciences du C.T.H.S. \#3 p. 37-53, 1981.

[BaFe] Helaman Ferguson and David Bailey, A polynomial time, numerically stable integer relation algorithm, RNR Technical Report RNR-91-032.

[Bak] A. Baker, Rational approximations to $2^{1 / 3}$ and other algebraic numbers, Quart. J. Math. Oxford (2) 15 (1964), 375-383.

[Ben] Michael Bennett, Effective measures of irrationality for certain algebraic numbers, J. Australian Math. Soc. (series A) 62 (1997), 329-344.

[Beu] Frits Beukers, A note on the irrationality of $\zeta(2)$ and $\zeta(3)$, Bull. London Math. Soc. 11 (1979), 268-272. Reprinted in [BerBoBo], 434-438.

[BerBoBo] Lennard Berggren, Jonathan Borwein, and Peter Borwein, "Pi: a source book", Springer, 1997.

[BoBo1] Jonathan Borwein and Peter Borwein, "Pi and the AGM", Wiley, 1987.

[BoBo2] Jonathan Borwein and Peter Borwein, More Ramanujan-type series for $1 / \pi$ in: "Ramanujan Revisited", Proceedings of the Centenary Conference, University of Illinois at UrbanaChampaign, June 1-5, 1987, edited by George E. Andrews et. al., Academic Press.

[BoBa] Jonathan Borwein and David Bailey, "Mathematics by experiment: plausible reasoning in the 21st century”, second edition, A.K. Peters/CRC Press, 2008.

[BoBaGi] Jonathan Borwein, David Bailey and Roland Girgensohn, "Experimentation in mathematics: computational paths to discovery", A.K. Peters/CRC Press, 2004.

[ChaS] Marc Chamberland and Armin Straub, Apéry limits: experiments and proofs, 6 Nov 2020. https://arxiv.org/abs/2011.03400

[Chu] G.V. Chudnowsky, On the method of Thue-Siegel, Ann. of Math. 117 (1983), 325-382.

[DKZ] Robert Dougherty-Bliss, Christoph Koutschan, and Doron Zeilberger, Tweaking the Beukers Integrals in search of more miraculous irrationality proofs À La Apéry, submitted. https://sites.math. rutgers.edu/ zeilberg/mamarim/mamarimhtml/beukers.html

[E1] D. Easton, Effective irrationality measures for certain algebraic numbers, Math. Comp. 46 (1986), 613-622.

[vdP] Alf van der Poorten, A Proof that Euler missed... Apéry's proof of the irrationality of $\zeta(3)$, Math. Intelligencer 1 (1979), 195-203. Reprinted in [BerBoBo], 439-447.

[V1] P. M. Voutier, Rational approximations to $2^{1 / 3}$ and other algebraic numbers revisited, Journal de Théorie des Nombres de Bordeaux 19 (2007), 265-288.

[V2] P. M. Voutier, Thue's Fundamentaltheorem, I, Acta Arith. 143 (2010), 101-144.

[Ze0] Doron Zeilberger, The method of creative telescoping, J. Symbolic Computation 11 (1991), 195-204. https://sites.math.rutgers.edu/ zeilberg/mamarimY/creativeT.pdf 
[Ze1] Doron Zeilberger, Closed form (pun intended!), in: "Special volume in memory of Emil Grosswald", M. Knopp and M. Sheingorn, eds., Contemporary Mathematics 143 579-607, AMS, Providence (1993). https://sites.math.rutgers.edu/ Zeilberg/mamarim/mamarimhtml/pun.html

[Ze2] Doron Zeilberger, Computerized deconstruction, Advances in Applied Mathematics 30 (2003), 633-654. https://sites.math.rutgers.edu/ zeilberg/mamarim/mamarimhtml/derrida.html

[Ze3] Doron Zeilberger, Book Review of F. Borwein and D. Bailey's Mathematics By Experiments and F. Borwein, D. Bailey and R. Girgensohn's Experimentation in Mathematics, American Scientist 93 \#2 (March/April 2005), 182-183. https://sites.math.rutgers.edu/ Zeilberg/mamarim/mamarimPDF/ mathexp.pdf

[Zu] Wadim Zudilin, Apéry-like difference equations for Catalan's Constant, https://arxiv.org/abs/ math/0201024 